\title{
Amino Acid Composition Reduces Frequency of Common Colds. Results of a Controlled Randomised Completely Masked Trial ${ }^{*}$
}

\author{
Alfons Meyer ${ }^{1}$, Doris Meister ${ }^{2 \#}$, Wilhelm Gaus ${ }^{3}$ \\ ${ }^{1}$ Kaiser-Friedrich-Ring 77, Wiesbaden, Germany; ${ }^{2}$ Medical Science Department, Kyberg Vital GmbH, Oberhaching, Germany; \\ ${ }^{3}$ Institute of Epidemiology and Medical Biometry, University of Ulm, Ulm, Germany. \\ Email: "Doris.Meister@kyberg.de
}

Received January $28^{\text {th }}, 2013$; revised February $28^{\text {th }}, 2013$; accepted March $7^{\text {th }}, 2013$

\begin{abstract}
Purpose: Special nutrients have an impact on common cold infections. In this trial we have investigated the effectiveness of the specific amino acid composition "aminoplus ${ }^{\circledR}$ immun" on the occurrence, duration and severity of common cold. Material and Methods: Our study is a controlled, randomised completely masked trial comparing genuine trial remedy versus placebo in parallel groups. Eligible subjects were people with recurrent and chronic infections of the ear, nose and pharyngeal area. The treatment period was 90 days. The primary outcome was the mean number of days that the patients had symptoms of infections. Results: From Oct. 2009 to Feb. 2010 a total of 145 patients were recruited, of whom 119 had no major protocol violation and were valid for assessment. Two thirds of the patients were female. The mean age of the patients was 45 years. $65 \%$ of participants had come into contact with many people and thereby an increased risk of infection. On average the patients had 11.1 infections during the 12 months before the study. Compliance for the intake of the study preparation was $98.9 \%$. Due to randomisation, the groups were very similar and balanced. The primary and all 6 secondary outcome variables showed a clear superiority of the investigated amino acid composition over placebo $(\mathrm{p}<0.0001)$. No intolerance was documented. Conclusion: The specific composition "aminoplus ${ }^{\circledR}$ immun is effective in preventing recurrent and chronic infections of the ear, nose and upper respiratory tract.
\end{abstract}

Keywords: Amino Acids; Immune System; Vitamins; Common Cold; Chronic Infection of Ear Nose; Pharyngeal Area

\section{Introduction}

Recurrent and chronic infections of the upper pharyngeal area are a common illness at all social levels and a frequent illness in the general practice. The causes of this infection are several viruses, such as adenoviruses, myxoviruses, corona viruses and picomaviruses. The human rhinovirus is by far the most common virus $(10 \%$ $25 \%$ ) and results in more medical consultations than any other viral or bacterial infection of the respiratory tract. These viral infections lead to a lack of productivity, restrictions in daily life and loss of social contact. Typically, recovery starts after 7 to 10 days. Possible complications are the extension of infection affecting the lower respiratory tract (e.g. bronchitis or pneumonia) or involvement of nasal ostia and Eustachian tubes. The mechanism through which virus infection triggers infec-

\footnotetext{
"The authors W.G. and A.M. declared that no competing interest exists. D.M. is employee of Kyberg Vital.

"Corresponding author.
}

tion of airway disease is still unclear, but likely to represent a constellation of host immune responses and viral action. Infection of the respiratory epithelium leads to a cascade of pro-inflammatory cytokines which further influence and activate immune cells of the airway tract [1, 2]. In general, treatment options for common cold ranges from anti-virus agents, acetylsalicylic acid and nutrients. The unfortunate reality is that common cold symptoms occur rather late during infection which often delays early diagnosis and effective therapy [3].

In this context the results of a survey on antibiotic use are interesting: in the German population only $41.4 \%$ of persons know, that antibiotics are not effective against common cold or influenza. Furthermore, $72.3 \%$ knew that antibiotics are effective against bacteria but not viruses $(52.6 \%)$ and $89 \%$ knew about antibiotic resistance [4].

Known nutrients believed to combat rhinovirus infections are vitamin $\mathrm{C}$, Echinacea and certain micronutrients which are found in a variety of cold drinks, where many 
of these contain further zinc or selenium. Vitamin C seems to be effective in reducing common cold symptoms if taken early, within $24 \mathrm{~h}$ of the onset of symptoms and vitamin $\mathrm{C}$ may be more effective in patients with a low level of vitamin $\mathrm{C}$ in general [2]. But overall there is no clear evidence about the single efficacy of vitamin $\mathrm{C}$ treatment of common cold, although vitamin $\mathrm{C}$ intake seems to be reasonable in cases of physical strain or insufficient intake [5]. Zinc acts as an essential cofactor in stimulating cytokine synthesis, and it is important for growth of fast proliferating immune cells and may protect immune cells from oxidative damage. Zinc deficiency is accompanied by a reduced synthesis of interferon gamma (IFN- $\gamma$ ), reduced activity of natural killer (NK) cells and a reduced T-cell activity [6]. Selenium acts as an essential cofactor for lymphocyte activity and protection of oxidative damage. A deficiency increases the synthesis of pro-inflammatory cytokines and leads to decreases in antibody generation and lymphocyte proliferation [6]. A deficiency of folic acid influences the activity of neutrophils, the cytotoxicity of T-Lymphocytes, antibody synthesis and the activity of NK cells. Similarly, a deficiency of vitamin B12, vitamin B6 or vitamin E can also influence and impair immune function [7]. As described different cells or mechanisms can be influenced by such nutrients. Generally, common cold, an infection of the upper respiratory system may be predisposed in a subject by an imbalanced or weak immune system. Therefore it can be hypothesized that the main target should be to improve and to stabilise the immune system, reducing inflammatory associated mediators, supporting the natural defence cells and barrier as already described in brief for vitamins and micronutrients. Besides these generally known nutrients used to combat rhinoviral infections, amino acids may serve in this context as a kind of adjunctive therapy. Therefore it is interesting to investigate the influence of a combined mixture of vitamins, trace elements and high dosage special immune system influencing amino acids, such as L-Glutamine, L-Arginine, L-Methionine, L-Lysine, L-Cysteine and Glycine and 2-Aminoethansulfonic acid, known as Taurine on such infections like common cold.

\section{Methods}

\subsection{Study Design}

This controlled randomised trial investigated the efficiency and safety of the specific nutrient composition (aminoplus ${ }^{\circledR}$ immun) for prevention of infections of ear, nose and pharyngeal area. The investigated remedy has been produced and distributed by Kyberg Vital GmbH in Germany. Kyberg Vital GmbH was also the sponsor of this study. The investigated product is a specially defined amino acid mixture with vitamins and trace elements (aminoplus ${ }^{\circledR}$ immun). The detailed composition is shown in Table 1.

This study was a randomised double blind trial with two parallel groups comparing genuine trial remedy versus a corresponding placebo. The placebo was composed of maltodextrine, sugar, acesulfam K, citric acid, colouring agent, isomalt, flavour, silicon dioxide, gum arabicum and contained no nutrients. It had the same flavour and texture as the genuine nutrient composition. The study protocol was according to current regulations [8] and signed between 10th July 2009 and 27th July 2009. The study was accepted by the Freiburg International Ethic Commission in D 79104 Freiburg on 12th Oct. 2009 and by the Ethic Commission of the Landesaerztekammer Hessen in D 60488 Frankfurt on 3rd Nov. 2009.

\subsection{Study Population}

Subjects eligible for the trial were women and men aged 18 to 70 years with at least two episodes of common cold and infections of ear, nose or pharyngeal area. Not eligible were people with severe infections like pneumonia, patients expecting an in-patient treatment during the trial, pregnant or breast feeding women, and people not able to

Table 1. Composition of the investigated amino acid composition (aminoplus ${ }^{\circledR}$ immun).

\begin{tabular}{cc}
\hline Nutrient & Daily dosage \\
\hline L-Glutamine & $3.000 \mathrm{mg}$ \\
L-Arginine & $2.000 \mathrm{mg}$ \\
L-Lysine & $1.000 \mathrm{mg}$ \\
Taurine & $1.000 \mathrm{mg}$ \\
Glycine & $700 \mathrm{mg}$ \\
L-Methionine & $500 \mathrm{mg}$ \\
Vitamin C & $300 \mathrm{mg}$ \\
L-Cysteine & $100 \mathrm{mg}$ \\
Magnesium & $100 \mathrm{mg}$ \\
Vitamin E & $30 \mathrm{mg}$ \\
Zinc & $10 \mathrm{mg}$ \\
Vitamin $B_{6}$ & $4.5 \mathrm{mg}$ \\
Vitamin $B_{2}$ & $4.2 \mathrm{mg}$ \\
$\beta$-Carotene & $2 \mathrm{mg}$ \\
Manganese & $2 \mathrm{mg}$ \\
Copper & $2 \mathrm{mg}$ \\
Folic acid & $1.2 \mathrm{mg}$ \\
Selenium & $0.1 \mathrm{mg}$ \\
Chromium & $50 \mu \mathrm{g}$ \\
Molybdenum & $50 \mu \mathrm{g}$ \\
Vitamin $\mathrm{B}_{12}$ & $9 \mu \mathrm{g}$ \\
\hline &
\end{tabular}


understand the protocol of the trial and treatment. Participants were recruited by four general practitioners, an alternative practitioner, or by placing an advert in local papers. All participants gave written informed consent. Participants received either three packages with genuine trial remedy or three packages with placebo. The placebo was identical in presentation and characteristics to the genuine trial remedy. A package contained 30 pouches with the study preparation. Every day, the participant had to put the content of one pouch in a glass of water, stir it and drink it. Hence, treatment duration was for 90 days. The randomisation was stratified for Hessen and Bavaria, which were the two areas where the study was performed. The randomisation code was produced with the computer program ROM at the Institute of Epidemiology and Medical Biometry at the Medical Faculty of the University of Ulm. The randomisation code list was always under the control of this institution. All packages of study preparation were labelled by W.G. None of the emergency envelopes to unmask the study was opened. Hence the study groups were completely masked to all investigators and subjects except W.G.

The participants in this trial had to fill in a case report form (CRF) at admission and at the end of the study. Furthermore, they filled in two CRF-pages of a diary on a daily basis. The diary questionnaire is shown in Table 2.

\subsection{Statistical Analysis}

The statistical analysis was undertaken by the SAS version 9.2. Distribution of the outcome variables is not known. Therefore, analysis of primary and secondary outcome variables was based on non-parametric rank- statistics. For statistical testing the Wilcoxon test for two parallel groups was employed.

\subsection{Outcome Measures}

For each day of the study participants recorded the following into the diary: their rectal temperature, whether they had symptoms of sneezing, a blocked nose or streaming/watering eyes, whether they had problems in the throat or in their swallowing, and whether they had a headache or pain in their arms or legs. These four criteria were put together with OR-operators as primary outcome. Secondary outcome variables were each of these symptoms separately and whether the patient felt fit and well.

Safety outcome: Any adverse reaction was queried and recorded every day. Moreover, the participants were asked in the diary about other medication taken apart from the study preparation and if she or he had consulted a physician.

\section{Results}

In total 145 patients were recruited between 22nd Oct. 2009 and 1st Feb. 2010. Treatment of the last patient was terminated on 1st May 2010. The data bank of the study was completed and closed on 9th Sep. 2010. Following completion of data collection, the randomisation code was added to the data bank. The biometrical report on the evaluation of the study was issued on 13th Oct. 2010. The package No. 105 of study preparation (placebo) was completely lost, and no patient identification is available. In the diaries of 25 patients $\leq 45$ days of treatment were documented. This was considered as a serious protocol violation because treatment duration should be more than half of the scheduled 90 days. Table 3 gives details of

Table 2. Diary questionnaire, to be filled by the patient every day of the study.

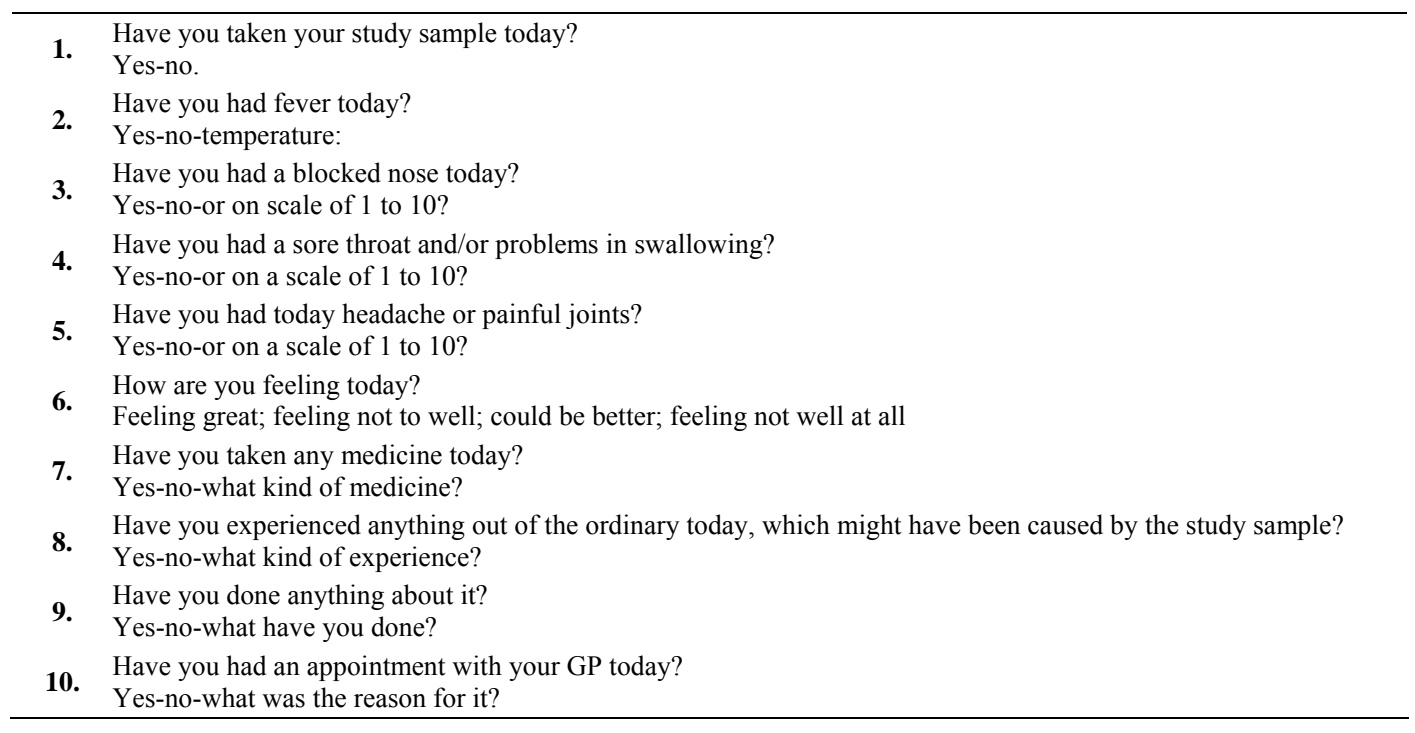


Table 3. Patients intended to treat (ITT) and patients treated according to protocol and therefore valid for effectiveness (VFE).

\begin{tabular}{|c|c|c|c|}
\hline & Genuine remedy & Placebo & Total \\
\hline Packages of Study preparation used = patients intended to treat $($ ITT) & 71 & 74 & 145 \\
\hline Packages lost & 0 & 1 & 1 \\
\hline Patients with incomplete treatment and/or incomplete documentation & 15 & 10 & 25 \\
\hline Patients treated according to protocol and valid for efficacy (VFE) & 56 & 63 & 119 \\
\hline
\end{tabular}

patients intended to treat (ITT) and patients treated according to protocol and therefore valid for efficacy (VFE) analysis. The scheduled treatment duration was 90 days. For the 119 VFE-patients $119 \times 90$ days $=10,710$ days of diary were planned. Actually, 11 patients did not fill in the diary completely and totally 24 days of diary were missing. Hence the documentation was $(10,710-24) / 10,710=99.8 \%$ complete.

In the study diary participants were asked if they had taken the study preparation every day of the study and the time of intake. The 119 VFE-patients recorded altogether 10587 days that they had taken the study preparation. The 99 days where this information was missing were counted as "no intake documented". Hence overall compliance for intake of study preparation is

$(10,587-99) / 10,710=98.9 \%$.

The 119 VFE-patients included 40 men and 79 women. They were between 18 and 70 years old, and on average 45 years. An increased risk for infections due to contact with many people was present in $65 \%$ of the patients. In the 12 months before the beginning of the study, the patients had an average of 11.1 infection episodes with an average duration of 9 days. At the beginning of the study, 105 patients took medication against an infection of the throat or nose. Column total of Table 4 gives some additional details of the study population.

Table 4 gives details of the two treatment groups at the beginning of the study. None of these variables are significantly different between the groups.

A day with an infection of the ear, nose or pharyngeal area was defined as a day with infection:

$=$ a day with fever;

OR symptoms of sneezing, a blocked nose or streaming/watering eyes;

OR problems in the throat or in swallowing;

OR headache or pain in arms or legs.

The number of patients with at least one infection during the study was defined as secondary outcome measure in the study. With genuine remedy, $86 \%$ of the patients had at least one episode of infection during the study, and with the placebo all patients had at least one episode of infection during the study.

The primary outcome was the number of days with infections during the 90 days of treatment. Patients with a genuine remedy had on average 16.9 days with infections and patients with the placebo had 38.4 days. The Wilcoxon test for two parallel groups computed a one sided $\mathrm{p}<0.0001$. In the study protocol a one sided level of significance was established with $2.5 \%$. Thus, the test is significant. More details on "effectiveness" are provided in Table 5.

To make sure that the patients with major protocol violations who were excluded from the "valid for efficacy" cohort of patients have not biased the results, an evaluation including these patients is necessary. The Intention to Treat (ITT) evaluation includes all 145 patients recruited for the study. However, therapeutic success is not known for the 26 patients with major protocol violations. For the purpose of evaluation and analysis, a poor therapeutic success is assumed for these 26 patients. The median and the Wilcoxon test for two parallel groups are based on ranks. Therefore, it does not matter how bad the success for the individual patient is as long as it is worse than the median. This technique is called "censored median" [9]. The censored median is 13.0 days with infection per patient during the 90 days of treatment for the group with genuine trial remedy and 41.0 days for the placebo group. For the ITT-evaluation the Wilcoxon test for two parallel groups computed a $p<0.0001$. There was a highly significant reduction of days with infections with the specific nutrient composition (aminoplus ${ }^{\circledR}$ immun) in the valid for efficacy (VFE)-cohort of patients as well as in the Intention to Treat (ITT)-cohort of patients.

All participants of the study were asked in the diary every day whether they had an adverse reaction, whether they had taken any medication in addition to the study preparation, and whether they had consulted a physician on that day. The results are presented in Table 6. Overall there is no evidence of intolerance or safety concerns of the investigated specific nutrient composition, which was well tolerated.

The concordance of all 6 secondary outcome variables shows the same superiority of the genuine trial remedy over the placebo. This is also true for the compounded primary outcome. Further, these results hold for the valid for efficacy (VFE) analysis as well as for the Intention to Treat (ITT) analysis including all recruited. 
Table 4. Description of patients and comparability of groups.

\begin{tabular}{|c|c|c|c|}
\hline & $\begin{array}{c}\text { Genuine remedy } \\
n=56\end{array}$ & $\begin{array}{c}\text { Placebo } \\
n=63\end{array}$ & $\begin{array}{c}\text { Total } \\
\mathrm{n}=119\end{array}$ \\
\hline Proportion of females & $71 \%$ & $62 \%$ & $66 \%$ \\
\hline Proportion of patients with contact with many people & $64 \%$ & $65 \%$ & $65 \%$ \\
\hline Mean number of infections in the 12 months before the beginning of the study & 12.2 & 10.2 & 11.1 \\
\hline Mean duration of these infections & 9.8 days & 8.7 days & 9.0 days \\
\hline Proportion of patients with a medication for an infection of the throat or nose at the beginning of the study & $88 \%$ & $89 \%$ & $88 \%$ \\
\hline Compliance of intake of study preparation & $98.9 \%$ & $98.9 \%$ & $98.9 \%$ \\
\hline
\end{tabular}

Table 5. Effectiveness-primary and secondary outcome.

\begin{tabular}{|c|c|c|c|}
\hline $\begin{aligned} \text { Primary outcome } \\
\end{aligned}$ & $\begin{array}{c}\text { Genuine remedy } \\
n=56\end{array}$ & $\begin{array}{c}\text { Placebo } \\
n=63\end{array}$ & $\begin{array}{c}\text { Total } \\
\mathrm{n}=119\end{array}$ \\
\hline Mean number of days with an infection (VFE) ${ }^{1}$ & 16.9 days & 38.4 days & 28.3 days \\
\hline $\begin{array}{l}\text { Median number of days with infection }{ }^{2} \\
\text { Valid for effectiveness (VFE) } n=56+63 \text { patients } \\
\text { Intended to treat (ITT) } n=71+74 \text { patients }\end{array}$ & $\begin{array}{l}9.1 \text { days } \\
13.0 \text { days }\end{array}$ & $\begin{array}{l}34.0 \text { days } \\
41.0 \text { days }\end{array}$ & $\begin{array}{l}19.0 \text { days } \\
30.0 \text { days }\end{array}$ \\
\hline Secondary outcomes & Genuine remedy & Placebo & Total \\
\hline Mean number of days with fever ${ }^{1}$ & 0.4 days & 4.1 days & 2.3 days \\
\hline Mean number of days with symptoms of sneezing, a blocked nose or streaming/watering eyes ${ }^{1}$ & 11.1 days & 29.9 days & 21.0 days \\
\hline Mean number of days with problems in the throat or to swallowing ${ }^{1}$ & 5.0 days & 17.0 days & 11.4 days \\
\hline Mean number of days with headache or pain in arms or legs ${ }^{1}$ & 8.1 days & 19.6 days & 14.2 days \\
\hline Proportion of days when the participant felt fit and well/healthy & $75 \%$ & $53 \%$ & $63 \%$ \\
\hline
\end{tabular}

${ }^{1}$ Mean number of days with the symptom per patient during the 90 days of treatment. This mean is based on all patients, not only on those who showed the symptom at least once during the study. Patients who never showed the symptom during the study are entered with 0 days; ${ }^{2}$ For patients with incomplete treatment and/or incomplete documentation the number of days with an infection is unknown. Therefore it is not possible to calculate the mean. But for these patients we assumed a poor result. Then the median is computed. For this censored median [9] it is sufficient to assume an outcome worse than the median.

Table 6. Tolerance and safety.

\begin{tabular}{|c|c|c|c|}
\hline & $\begin{array}{c}\text { Genuine } \\
\text { remedy } \\
\mathrm{n}=56\end{array}$ & $\begin{array}{c}\text { Placebo } \\
n=63\end{array}$ & $\begin{array}{c}\text { Total } \\
\mathrm{n}=119\end{array}$ \\
\hline Number of patients with at least one adverse reaction during study & 7 patients & 14 patients & 21 patients \\
\hline Total number of days with an adverse reaction & 29 days & 46 days & 75 days \\
\hline Total number of serious adverse reaction & 0 & 0 & 0 \\
\hline Proportion of patients with at least one intake of medication other than the study preparation during the study & $64 \%$ & $98 \%$ & $82 \%$ \\
\hline Mean duration of intake of these medications & 28.6 days & 31.9 days & 30.7 days \\
\hline Proportion of patients with at least one visit to a physician & $39 \%$ & $44 \%$ & $55 \%$ \\
\hline Total number of visits to a physician & 63 visits & 110 visits & 173 visits \\
\hline
\end{tabular}

\section{Discussion}

The results of this present randomised controlled study shows evidence that the specific nutrient composition (aminoplus ${ }^{\circledR}$ immun) has an impact on the occurrence and duration of infections of the ear, nose and pharyngeal, which are usually known as common cold. In this noninvasive study, there was no focus on looking at function of immune parameters as a physiological response due to nutrient intake. Although amino acids have multifunctional properties, especially on immune modulation as already described, it is impossible to define one or two single parameters as a "golden standard" for measurement of immune function. Due to this, we have developed a standardised clinical questionnaire and report on a controlled, randomised trial with blinded treatment groups.

The participants who took the special nutrient composition had significantly less days of infection compared with the placebo. Furthermore, the patients with the genuine trial remedy have had reduced days of fever, reduced days of cold symptoms and reduced days with problems in swallowing due to a throat infection. The improved 
outcome of patients who had taken the genuine remedy can be explained by the variety of immune modulating influence of amino acids along with vitamins and micronutrients. Whether the observed effectiveness is driven mainly by amino acids can be open to speculation. However, amino acids are nutrients with multiple functions in the body and also with specially defined functions on the immune system. The amino acid Glutamine has been shown in several in vitro and in vivo studies, summarized by Li et al. [10], having an impact on lymphocyte proliferation in response to stimulation by T-cell mitogens. Glutamine seems to prevent apoptosis, stimulate cell growth and promote antibody production in lymphocytes. Apart from these, Glutamine has a further impact on several immunological activities [10]. The alkaline amino acid Arginine stimulates $\mathrm{T}$ lymphocytes in response to mitogens and helps to kill tumour cells by activated macrophages. High concentration of Arginine seems to increase the cytotoxicity of monocytes and NK cells in vitro [10]. Arginine demonstrates in this context a variety of therapeutical effects. An oral Arginine supplementation increases peripheral blood lymphocyte mitogenesis, T-helper to T-cytotoxic cell ratio, macrophage activity against microorganisms and tumour cells and decrease Tc cells [11]. These amino acids can influence immune cells in respect to Glutamine as energy substrate for leucocytes, serve as a major fuel for enterocytes and are necessary for the proliferation of lymphocytes in response to stimulation by T-cell mitogens. Arginine has been shown to have an influence on T-cell function, antibody production and the accelerating of wound healing. The amino acid Lysine has shown a major impact on viral infections, particularly in Herpes simplex labialis infections [10].

Sulphur containing amino acids like Methionine, Cysteine and 2-Aminoethansulfonic acid are not only influencing different metabolic steps, as in the case of Methionine [12] but also several immune functions. Sulphur containing amino acids, like Cysteine, can be derived from the essential amino acid Methionine or via diet. Cysteine is the rate-limiting amino acid in the synthesis of intracellular Glutathione. Depletion of Glutathione results in increased vulnerability to oxidative stress [13]. Oxidative stress and inflammation are well-recognized conditions. During infection and injury there is a higher demand in Cysteine. Lyer et al. [14] have shown that plasma Cysteine in humans is negatively associated with plasma IL-1 beta levels. This shows that a nutritional supplementation with Cysteine or its precursor during early infection may be an intervention to lessen acute inflammation and accompanied tissue injury. On reviewing these different effects of immune system modulation by nutrients, it seems to be a logical consequence, that this special defined oral application of amino acids and nutrients has an immune modulation influence and therefore a measurable impact on infections collectively known as common cold.

Amino acids are not only relevant in common cold disease but also other human diseases. Immune modulating amino acids, as in the case of glutamine, also play an interesting role in inflammatory bowel disease (IBD). This amino acid is generally a major ingredient of formula diet to improve nutritional status and to influence the intestinal system. For example in IBD glutamine acts as an additional therapeutic component. IBD is characterised by chronic intestinal inflammation, possibly related to an imbalance of immune response to luminal factors. Glutamine improves the intestinal barrier function and stimulates intestinal epithelial cell proliferation (IEC) and reduces IEC apoptosis. Glutamine is able to modulate inflammatory and oxidative response and in vitro data indicate that cytokine production by macrophages is influenced by glutamine [15]. Apart from glutamine, sulphur containing amino acids are important for immune function as these are precursors of glutathione [16]. These amino acids containing sulphur contribute to the maintenance and integrity of the cellular system by for example influencing the cellular redox state, detoxifying toxic compounds and influencing free radicals and reactive oxygen species [17]. In recent years several animal and clinical studies have investigated the influence of nutrients on immune parameters. Vitamin $\mathrm{C}$ serves as a scavenger for reactive oxygen species, generated by activated immune cells. Vitamin $\mathrm{C}$ is also highly concentrated in leucocytes and rapidly used up during infections. Several B-vitamin deficits may result in an impairment of Lymphocyte maturation and growth. Folic acid acts closely in cooperation with vitamin B6 and vitamin B12 in the synthesis of nucleic acids and protein. A deficiency of folic acid alters immune competence and resistance to infections. It therefore influences the cell mediated immunity by reducing the proportion of circulation T-Lymphocytes and their response to mitogen activation. Selenium plays a key role in antioxidants function and redox regulation via glutathione peroxidise. The well-known micronutrient zinc has an influence on innate and acquired immune functions [18].

The outcome of this study shows a significant effect in preventing infections of recurrent and chronic infections of the ear, nose and pharyngeal area. The investigated specific composition (aminoplus ${ }^{\circledR}$ immun), composed of immune competent amino acids along with vitamins and micronutrients, has a major impact on influencing and boosting the immune system. Although the impact of aminoplus ${ }^{\circledR}$ immun might be a matter of speculation, the immune influencing impact of nutrients cannot be un- 
derestimated. All nutrients are available in an easily accessible form and the results have shown a positive effect on health in common cold.

\section{Conclusion}

This controlled, randomised, double-blind study compared the defined nutrient composition "aminoplus ${ }^{\circledR}$ immun" in oral application versus a placebo. The result of this study shows a significant efficacy in reducing severity and duration of recurrent and chronic infections of the ear, nose and pharyngeal area, usually described as common cold. This amino acid composition is a safe and well tolerated formula. Further studies ought to look deeper into such a composition in medical practice as a safe and easily administered therapeutic option. Nutritional therapies are often of major interest to general practitioners, dietician as well as patients. Results of controlled clinical trials are the main proof for effectiveness of a specific therapy, but surveys and epidemiological data should also be taken into account for new strategies in medical and nutritional therapy.

\section{Author's Contribution}

W.G., A.M. and D.M. designed the study. A.M. recruited patients. Statistical analysis was performed by W.G. W.G. and D.M. contributed to the interpretation of the data and drafting of the manuscript along with A.M. All authors agreed on the final version of the manuscript.

\section{Acknowledgements}

Primarily we want to thank all participants of the study for their cooperation and their patience in filling in so many pages in the diary. At the Institute of Epidemiology and Medical Biometry of the University of Ulm Johanna Ballasch and Stella Orlogi created the study data bank. Silvia Sander and Johanna Ballasch undertook the SAS programming for the evaluation of the study. Many thanks for their excellent work.

\section{REFERENCES}

[1] J. Footitt and S. L. Johnston, "Cough and Viruses in Airways Disease: Mechanism," Pulmonary Pharmacology \& Therapeutics, Vol. 22, No. 2, 2009, pp. 108-113. doi:10.1016/j.pupt.2008.12.022

[2] D. Kilgore and W. Najm, "Common Respiratory Diseases," Primary Care Clinical Office Practise, Vol. 37, No. 2, 2010, pp. 297-324.

[3] G. Rohde, "Drug Targets in Rhinoviral Infections," Infectious Disorders-Drug Targets, Vol. 9, No. 2, 2009, pp. 126-132.

[4] M. S. Faber, K. Heckenbach, E. Velasco and T. Eck- manns, "Antibiotics for the Common Cold: Expectations of Germany's General Population," Research Article, 2010, pp. 1-7. www.eurosurveillance.org

[5] A. Ströhle and A. Hahn, "Vitamin C and Immune Function," Medizinische Monatsschrift Pharmacologie, Vol. 32, No. 2, 2009, pp. 49-54.

[6] P. Stehle, "Immunonutrition-Nutrients with Immunomodulating Action, Part 1: Amino Acids," ErnährungsUmschau, Vol. 47, No. 6, 2000, pp. 216-222.

[7] A. Ströhle and A. Hahn, "Bedeutung von Mikronährstoffen für die Immunkompetenz," MMW-Fortschritte der Medizin Orginalien, No. 3, 2009, pp. 133-141.

[8] W. Gaus and D. Chase, "Klinische Studien: Regelwerke, Strukturen, Dokumente, Daten," 2nd Edition, Verlag, Norderstedt, 2008.

[9] E. M. Lemmel, M. Franke, W. Gaus, P. W. Hartl, P. H. Hofschneider, K. Miehlke, K. Machalke and H. J. Obert, "Results of a Phase-II Clinical Trial on Treatment of Rheumatoid Arthritis with Recombinant Interferon-Gamma," Rheumatology International, Vol. 7, No. 3, 1987, pp. 127-132. doi:10.1007/BF00270465

[10] P. Li, Y.-L. Yin, D. Li, S. W. Kim and G. Wu, “Amino Acids and Immune Function," British Journal of Nutrition, Vol. 98, No. 2, 2007, pp. 237-252. doi:10.1017/S000711450769936X

[11] M. A. Potenza, C. Nacci and D. Mitola-Chippa, "Immuno-Regulatory Effects of L-Arginine and Therapeutic Implications," Current Drug Targets-Immune, Endocrine \& Metabolic Disorders, Vol. 1, No. 1, 2001, pp. $67-$ 77. doi:10.2174/1568008013341811

[12] J. T. Brosnan and M. E. Brosnan, "The Sulphur-Containing Amino Acids: An Overview," Journal of Nutrition, Vol. 136, No. 6S, 2006, pp. 1636S-1640S.

[13] G. Atmaca, "Antioxidant Effects of Sulphur-Containing Amino Acids," Yonsai Medical Journal, Vol. 45, No. 5, 2004, pp. 776-788.

[14] S. S. Iyer, C. J. Accardi, T. R. Ziegler, R. A. Blance, J. D. Ritzenthaler, M. Rojas, J. Roman and D. P. Jones, "Cysteine Redox Potential Determines Pro-Inflammatory Il-1b levels," PLoS One, Vol. 4, No. 3, 2009, pp. 1-10.

[15] M. Coeffier, R. Marian-Letellier and P. Dechelatte, "Potential of Amino Acids Supplementation during Inflammatory Bowel Disease," Inflammatory Bowel Disease, Vol. 16, No. 5, 2010, pp. 18-24.

[16] R. F. Grimble "The Effects of Sulphur Amino Acid Intake on Immune Function in Humans," Journal of Nutrition, Vol. 136, No. 6, 2006, pp. 1660S-1665S.

[17] M. E. Nimni, B. Han and J. Cordoba, "Are We Getting Enough Sulphur in Our Diet?" Nutrition \& Metabolism, Vol. 4, No. 24, 2007, pp. 1-12. doi:10.1186/1743-7075-4-24

[18] S. Maggin, E. S. Wintergerst, S. Beveridge and D. H. Hornig, "Selected Vitamins and Trace Elements Support Immune Function by Strengthening Epithelial Barriers and Cellulare and Humorale Immune Response," British Journal of Nutrition, Vol. 98, No. 1, 2007, pp. S29-S35. 


\section{Abbreviations}

CRF: case report form; ITT: intended to treat; VFE: valid for efficacy. 